\title{
LOCAL METHOD OF APPROXIMATE PARTICULAR SOLUTIONS FOR TWO-DIMENSIONAL UNSTEADY INCOMPRESSIBLE FLOW
}

A meshless local method of approximated particular solutions (LMAPS) is used to analyze incompressible fluid flow in a two dimensionalcavity. The method solves the incompressible Navier-Stokes equations in terms of theprimitive variables using the fractional step scheme. The basic equations are derived via interpolation using integrated multiquadrics radial basis functions. Lid-driven cavity benchmark case for various Reynolds numbers is presented in the article. The procedure produces stable solutions with results comparable to those in literature.

Keywords: Meshless, localized method of approximated particular solutions, incompressible flows, Navier-Stokes equations.

\section{Introduction}

The isothermal incompressible viscous flow is used to describe flows taking advantage of simplified flow equations that describe fluid motion, which is known as the incompressible Navier-Stokes equations. There are numerous approaches developed to solve the incompressible Navier-Stokes equations; the algorithm used in this article represents the Chorin's projection method (fractional step) that belongs to the class of the decoupled methods which are more commonly used in computational fluid dynamics (CFD). Other way to classify the algorithms is to determine the version of the underlying governing equations, whether they are expressed using the primitive variables, velocity component and pressure, or not [1]. The mentioned fractional step method uses primitive variable formulation of the Navier-Stokes equations.

The fractional step algorithm is chosen to approximate the solutions of the incompressible Navier-Stokes equations in conjunction with the localized method of approximated particular solutions (LMAPS) that represents meshless collocation method [2].

The fractional step method solves the incompressible NavierStokes equations in three steps. In the first step the simple form of the momentum equations is obtained by dropping the pressure source term; this results in the intermediate velocities. In the next step the intermediate velocities are projected on the divergence-free plane using the solution of the Poisson's pressure equation and, subsequently obtaining velocity corrections using the pressure gradients [1]

The main drawback of mesh-based numerical methods is the requirement of the mesh generation, which may be difficult to process during the numerical implementations, especially for multi-dimensional problems. In order to avoid mesh generation process many meshless numerical methods, such as the local boundary integral element method (LBIEM) [3 - 7], smoothed particle hydrodynamics (SPH) method [8], meshless strong (collocation) method [9 - 11] and meshless local Petrov-Galerkin method (MLPG) [12 and13] have been developed. The LMAPS used in this study represents a stable, accurate tool for simulating the two-dimensional incompressible viscous flow field with the projection method. Numerical experiment of the two-dimensional lid-driven cavity flow problem is presented in the article with the comparison against literature data [14], which verifies the performance of the LMAPS.

\section{Governing equations}

An unsteady incompressible flow is governed by NavierStokes equations, which can be in a two dimensional case written in dimensionless form using the primitive variables as

$$
\begin{aligned}
& \frac{\partial u}{\partial t}+u \frac{\partial u}{\partial x}+v \frac{\partial u}{\partial y}=\frac{1}{\operatorname{Re}}\left(\frac{\partial^{2} u}{\partial x^{2}}+\frac{\partial^{2} u}{\partial y^{2}}\right)-\frac{\partial p}{\partial x} \\
& \frac{\partial v}{\partial t}+u \frac{\partial v}{\partial x}+v \frac{\partial v}{\partial y}=\frac{1}{\operatorname{Re}}\left(\frac{\partial^{2} v}{\partial x^{2}}+\frac{\partial^{2} v}{\partial y^{2}}\right)-\frac{\partial p}{\partial y} \\
& \frac{\partial u}{\partial x}+\frac{\partial v}{\partial y}=0
\end{aligned}
$$

\footnotetext{
* Juraj Muzik, Ladislav Kais, Roman Bulko

Department of Geotechnics, Faculty of Civil Engineering, University of Zilina, Slovakia

E-mail: juraj.muzik@fstav.uniza.sk
} 
where $u, v$ is the velocity vector component in the direction $x$ and $y$ respectively, $p$ is the pressure, and Re represents Reynolds number, assuming that density and characteristic velocity equals to unity. A fractional step algorithm is used to solve this problem (see [1, 15 and 16]). The time derivative of the velocity in momentum equations Eq. $(1,2)$ can be replaced with a difference and the following relation is obtained:

$$
\begin{aligned}
& u^{n+1}=u^{n}+\Delta t\left[\frac{1}{\operatorname{Re}}\left(\frac{\partial^{2} u^{n}}{\partial x^{2}}+\frac{\partial^{2} u^{n}}{\partial y^{2}}\right)-\right. \\
& \left.-u^{n} \frac{\partial u^{n}}{\partial x}-v^{n} \frac{\partial u^{n}}{\partial y}-\frac{\partial p^{n+1}}{\partial x}\right] \\
& v^{n+1}=v^{n}+\Delta t\left[\frac{1}{\operatorname{Re}}\left(\frac{\partial^{2} v^{n}}{\partial x^{2}}+\frac{\partial^{2} v^{n}}{\partial y^{2}}\right)-\right. \\
& \left.-u^{n} \frac{\partial v^{n}}{\partial x}-v^{n} \frac{\partial v^{n}}{\partial y}-\frac{\partial p^{n+1}}{\partial x}\right]
\end{aligned}
$$

where upper indexes $n$ and $n+1$ indicate time level and $\Delta \mathrm{t}$ is the length of the timestep. The first order finite difference, used here to discretize unsteady term, is preffered beacuse the explicit nature of the momentum equations. Eq. $(4,5)$ arean explicit formula for the convection andviscous terms, and an implicit one for the pressure term. Eq. $(4,5)$ are simplified using the fractional time step approximation (e.g., [15 and 16]), which computes the intermediate velocity $\tilde{u}, \tilde{v}$ using the simplified momentum equation

$\tilde{u}=u^{n}+\Delta t\left[\frac{1}{\operatorname{Re}}\left(\frac{\partial^{2} u^{n}}{\partial x^{2}}+\frac{\partial^{2} u^{n}}{\partial y^{2}}\right)-u^{n} \frac{\partial u^{n}}{\partial x}-v^{n} \frac{\partial u^{n}}{\partial y}\right]$

$\tilde{v}=v^{n}+\Delta t\left[\frac{1}{\operatorname{Re}}\left(\frac{\partial^{2} v^{n}}{\partial x^{2}}+\frac{\partial^{2} v^{n}}{\partial y^{2}}\right)-u^{n} \frac{\partial v^{n}}{\partial x}-v^{n} \frac{\partial v^{n}}{\partial y}\right]$

Comparing $(4,5)$ and $(6,7)$ respectively gives

$u^{n+1}=\tilde{u}-\Delta t \frac{\partial p^{n+1}}{\partial x}$

$v^{n+1}=\tilde{v}-\Delta t \frac{\partial p^{n+1}}{\partial x}$

The intermediate velocity components $\tilde{u}, \tilde{v}$ do not satisfy the continuity equation in (3). The velocity components $u^{\mathrm{n}+1}, v^{\mathrm{n}+1}$ must satisfy the continuity equation, which implies

$\frac{\partial}{\partial x}\left(\tilde{u}-\Delta t \frac{\partial p^{n+1}}{\partial x}\right)+\frac{\partial}{\partial y}\left(\tilde{v}-\Delta t \frac{\partial p^{n+1}}{\partial y}\right)=0$

A pressure Poisson equation then results directly from equation (10)

$\frac{\partial^{2} p^{n+1}}{\partial x^{2}}+\frac{\partial^{2} p^{n+1}}{\partial y^{2}}=\frac{1}{\Delta t}\left(\frac{\partial \tilde{u}}{\partial x}+\frac{\partial \tilde{v}}{\partial y}\right)$

\section{Localized method of approximated particular solutions}

In order to apply the LMAPS, the following procedure may beadopted to solve physical variable $u$ with any given differential operator.Even though the implementation details of the LMAPS are quite similar to the meshless collocation methods, its origin follows the globally defined method of approximated particular solution (MAPS) (see [2]). In this study the LMAPS is treated as special case of the meshless collocation methodwith the support domain concept already included in the formulation.

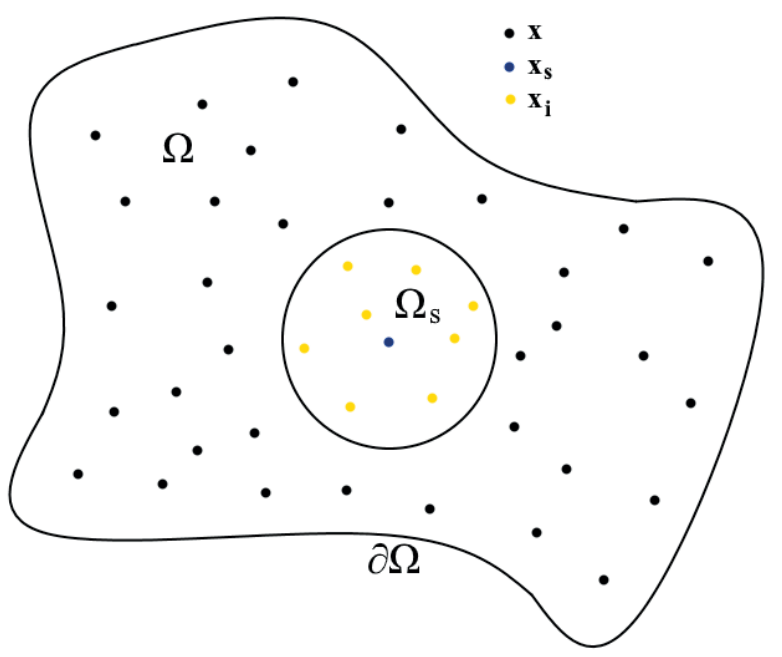

Fig. 1 The diagram of global domain $\Omega$, local support domain $\Omega$ of point $x_{s}$ global points xand local point $x_{i}$

The area of interest $\Omega$ with the boundary $\partial \Omega$ is covered by points within the area and also on the global boundary (see Fig. 1). Consider a local circular sub-domain $\Omega_{s}$ centered at every point $s$. This sub-domain is regular around all the points, and it is called support domain. Then using the points in a particular support domain any function can be expressed using just nodal values as

$u(\mathbf{x})=\sum_{i=1}^{N S} \alpha_{i} F\left(\left\|\mathbf{x}-\mathbf{x}_{i}\right\|\right), x \in \Omega$

where $N S$ is the total number of computational nodes in the support domain, $F$ is the radial basis function, $\mathbf{x}$ is coordinate vector and $\alpha_{i}$ are the local weighting coefficients to be determined. Because the values of $u$ expressed using Eq. (12) at the computational nodes should be the same as $u$ specified in these nodes, we can write following matrix expression

$\mathbf{u}=\mathrm{F} \alpha$

where

$\mathbf{u}_{i}=\left[\begin{array}{llll}u\left(\mathbf{x}_{1}\right) & \ldots & u\left(\mathbf{x}_{N S}\right)\end{array}\right]^{T}$ 
$\mathbf{F}=\left[\begin{array}{ccc}F\left(\left\|\mathbf{x}_{1}-\mathbf{x}_{1}\right\|\right) & \cdots & F\left(\left\|\mathbf{x}_{1}-\mathbf{x}_{N S}\right\|\right) \\ \vdots & \ddots & \vdots \\ F\left(\left\|\mathbf{x}_{N S}-\mathbf{x}_{1}\right\|\right) & \cdots & F\left(\left\|\mathbf{x}_{N S}-\mathbf{x}_{N S}\right\|\right)\end{array}\right]$

$\alpha=\left[\begin{array}{lll}\alpha_{1} & \cdots & \alpha_{N S}\end{array}\right]^{T}$

Since $\mathbf{F}$ is invertible, we will obtain the unknown weighting coefficients as

$\alpha=\mathrm{F}^{-1} \mathbf{u}$

Now, for operators concerned in solving Navier-Stokes equations, we firstly consider the operator with highest order, the Laplace operator $\nabla^{2}$, and let

$\nabla^{2} u\left(\mathbf{x}_{i}\right)=\sum_{i=1}^{N S} \alpha_{i} f\left(\left\|\mathbf{x}-\mathbf{x}_{i}\right\|\right)=\mathbf{f} \alpha$

where $f$ is the radial basis function with the essential condition as $f=\nabla^{2} F$. Replacing $\alpha_{i}$ with Eq. (17) we get

$\nabla^{2} u(\mathbf{x})=\mathbf{f F}^{-1} \mathbf{u}=\varphi \mathbf{u}$

where $\varphi$ is the vector of shape functions Laplacian. We apply similar procedures to the gradient operator $\nabla$ and we get

$\nabla_{j} u\left(\mathbf{x}_{i}\right)=\sum_{i=1}^{N S} \alpha \frac{\partial F\left(\left\|\mathbf{x}-\mathbf{x}_{i}\right\|\right)}{\partial x_{j}}=\mathbf{G}_{, j} \alpha=$

$=\mathrm{G}_{, j} \mathrm{~F}^{-1} \mathbf{u}=\gamma \mathbf{u}$

where $\gamma$ is the vector of shape function gradients and subscript $j$ represents the coordinate of the gradient.

The processed operators may now be applied directly into numerical implementations, which discretizes the governing equations and the essential conditions into formulation of linear systems. All boundary conditions in this paper have also been processed using the LMAPS. Numerical procedures for solving Navier-Stokes equations will be elaborated in subchapter 3.3, and more details of the LMAPS can be found in Ref. [2].

\subsection{Integrated multiquadrics radial basis functions}

The trial function basis used in the LMAPS incorporates multiquadrics RBF, but different approach than the standard meshless collocation methods is adopted. In the case of LMAPS the MQ-RBF [2 and 17] represents the form of basis function after differentiation using highest order operator. To obtain the basis function, MQ-RBF needs to be integrated.
In this article the two-dimensional integrated multiquadrics radial basis function (integrated MQ-RBF) is used as the basis function $F$. The MQ-RBF

$f(r)=\sqrt{r^{2}+c^{2}}$

and the integrated MQ-RBFs [2]

$F(r)=\frac{1}{9}\left(r^{2}+4 c^{2}\right) \sqrt{r^{2}+c^{2}}-\frac{1}{3} c^{3}$

$\ln \left(\sqrt{r^{2}+c^{2}}+c\right)$

wherer depicts the distance between the collocation points and $c$ is a shape parameter.

\subsection{Handling derivative boundary conditions}

Strong-form methods can produce accurate results for partial differential equations, when the boundary conditions are all of Dirichlet type. If there is any derivative boundary condition, the accuracy of the solution deteriorates drastically, and the solution can be unstable; small changes in the setup of the problem can lead to a large change in the solution. The discretized

system equation behaves like an ill-posed problem in which errors introduced into the system are magnified in the output. A number of strategies can be used to impose the derivative boundary conditions in the strong form methods. The method using fictitious points is used in this article to enforce the pressure gradient on the boundary.

Along the derivative boundaries, a set of fictitious points is added outside the problem domain. In this case, two sets of equations are established at each derivative boundary node, one for the derivative boundary condition, and the other for the governing equation.

\subsection{Discretization of Navier-Stokes equations}

In this section, the numerical procedures for the approximation of the Navier-Stokes equations via the fractional step method and the LMAPS will be explained. The fractional step begins with Eq. $(6,7)$, where the intermediate velocity can be obtained for $i$ th node explicitly as follows:

$\tilde{u}_{i}=u_{i}^{n}+\Delta t\left[\frac{1}{\operatorname{Re}} \varphi_{i} \mathbf{u}_{i}^{n}-u_{i}^{n} \gamma_{i, x} \mathbf{u}_{i}^{n}-v_{i}^{n} \gamma_{i, y} \mathbf{u}_{i}^{n}\right]$
$\tilde{v}_{i}=v_{i}^{n}+\Delta t\left[\frac{1}{\operatorname{Re}} \varphi_{i} \mathbf{v}_{i}^{n}-u_{i}^{n} \gamma_{i, x} \mathbf{v}_{i}^{n}-v_{i}^{n} \gamma_{i, y} \mathbf{v}_{i}^{n}\right]$

The boundary conditions of intermediate velocity are given as the same as the boundary conditions of the original velocity in 
the present study. Next, we obtain pressure term from Poisson's equation of the second stage, which can be written as

$\varphi \mathbf{p}^{n+1}=\frac{1}{\Delta t}\left(\gamma_{x} \tilde{\mathbf{u}}+\gamma_{y} \tilde{\mathbf{v}}\right)$

Then, in the third stage we execute the velocity correction explicitly as

$u_{i}^{n+1}=\tilde{u}_{i}-\Delta t \gamma_{i, x} \mathbf{p}^{n+1}$

$u_{i}^{n+1}=\tilde{v}_{i}-\Delta t \gamma_{i, y} \mathbf{p}^{n+1}$

The convergence criterion for steady state is given as $\varepsilon=10^{-7}$ for all numerical experiments in this paper. The fractional time step algorithm described above is now used to solve the NavierStokes equations at every time step [15]:

- Step 1: Computation of the intermediate velocities $\tilde{u}, \tilde{v}$ from the velocities at the previous time step using $(23,24)$.

- Step 2: Solution of pressure Poisson equation (25).

- Step 3: Computation of velocities at the current time step from $(26,27)$.

\section{Numerical example}

The solution of the Navier-Stokes equations using the LMAPS developed in the previous sections is validated here by solving lid-driven cavity flow examples. Lid-driven cavity flow is used as a standard test problem for the validation of numerical solutions of incompressible Navier-Stokes flow. The top wall of the cavity moves with a velocity $u_{0}=1$, and no-slip impermeable boundary conditions are assumed along the other three walls. The geometry and boundary conditions arepresented in Fig. 2. The difficulty of this problem lies in the presence of singularities of pressure and velocity at the two upper corners of the cavity [15]. Therefore, the density of points used increases toward the corners of the cavity (see Fig. 2).

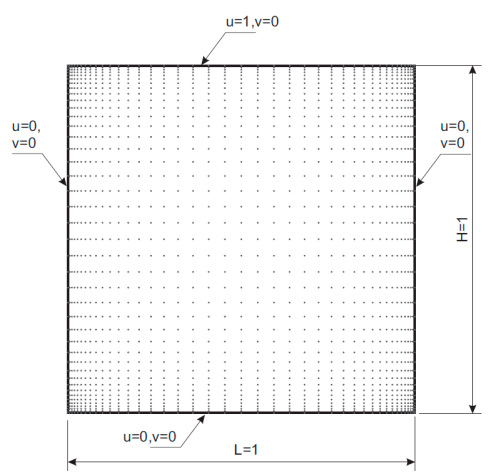

Fig. 2 Lid-driven cavity, boundary conditions, $41 \times 41$ points distribution
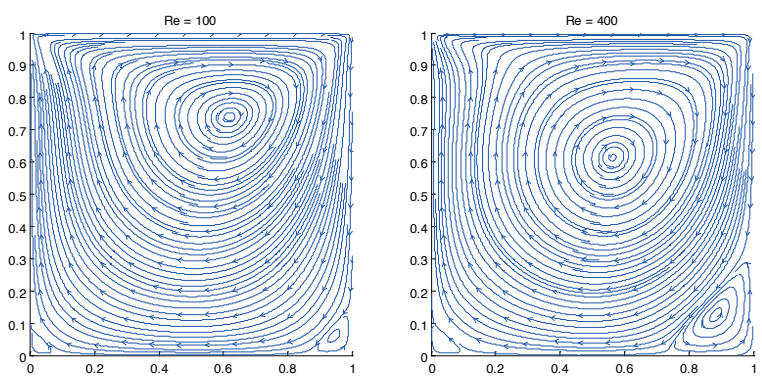

$\mathrm{Re}=1000$

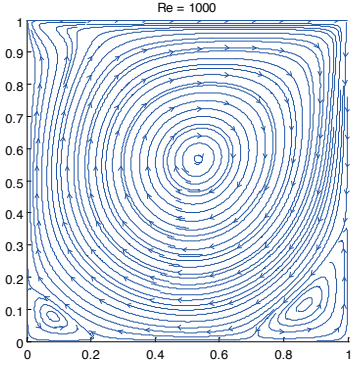

$\mathrm{Re}=5000$

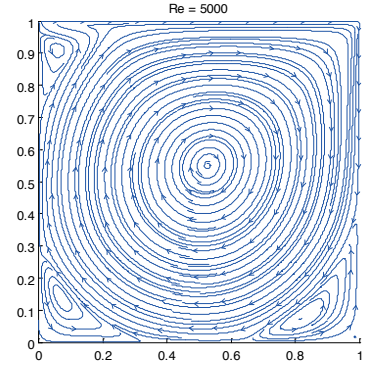

Fig. 3 Lid-driven cavity, streamlines pattern for different Reynolds numbers

Three different meshes are tested here $(61 \times 61,81 \times 81$, and $101 \times 101$ points), with each having a non-uniform distribution similar to that used in [15]. For all calculations the uniform timestep $\Delta \mathrm{t}=10^{3}$. The strategy of finding neighboring points for $\mathrm{RBF}$ interpolation is the main problem facing the use of anonuniform distribution of points.

The segmentation strategy (see [7]) gives the best results for this type of network. For higher Reynolds numbers the solution found using a coarse network does not converge and denser networks must be used. This problem probably arises because the point distribution in the coarse network is unable to capture the velocity gradient within a thin boundary layer for higher Reynolds numbers. The results of the LMAPS are compared with the solutions presented in [14] for the Reynolds number $\operatorname{Re}=100,400,1000$ and 5000. The vertical velocity components along a horizontal line through the center ofthe cavity and the horizontal velocity components along a vertical line through the center of the cavity are plotted in Fig. 4.

These comparisons for the three types of mesh show that, as expected, the most dense mesh $(101 \times 101$ points $)$ leads to the results closest to those of [14]. Figures 3, 5 and 6 present streamlines, velocity magnitude and pressure contours for Reynolds numbers $\mathrm{Re}=100,400,1000$ and 5000 .

\section{Conclusions}

A possible use of the localized method of approximate particular solutions with integrated MQ-RBF interpolation 

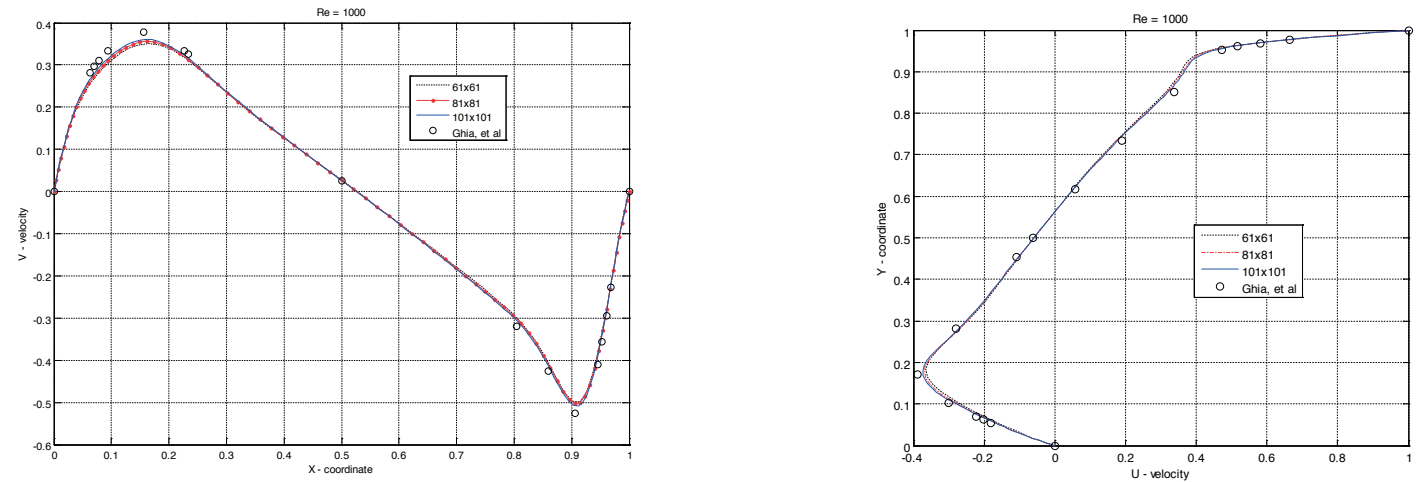

Fig. 4 Lid-driven cavity, $R e=1000$,vertical velocity components along horizontal line $y=0.5$ and horizontal velocity components along vertical line $x=0.5$
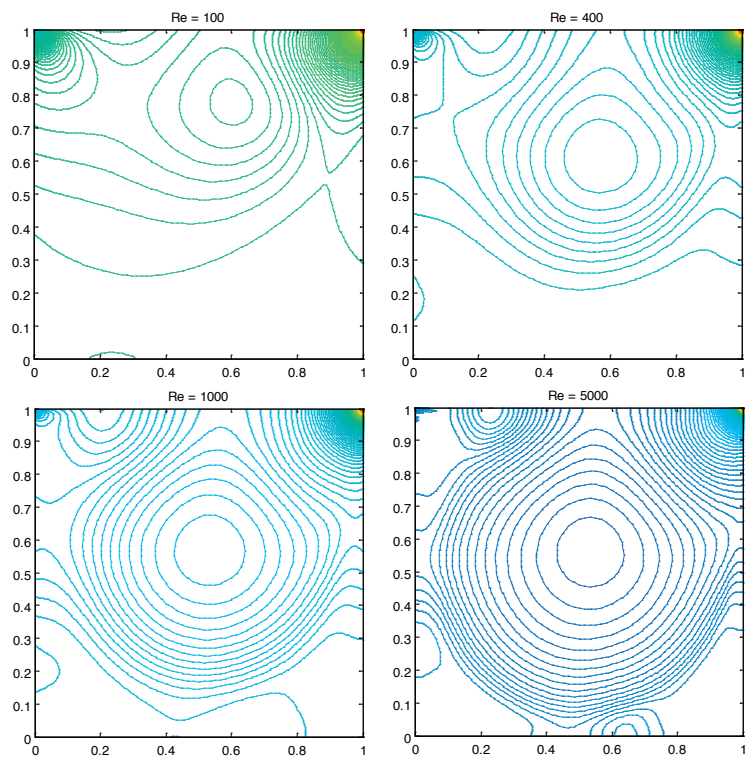

Fig. 5 Lid-driven cavity, pressure contours for different Reynolds numbers

ispresented here for the computation of incompressible flows The primitive variable formulation of the Navier-Stokes equations and the fractional step scheme is used to achieve stable and accurate results. The suitability of this procedure is tested in the
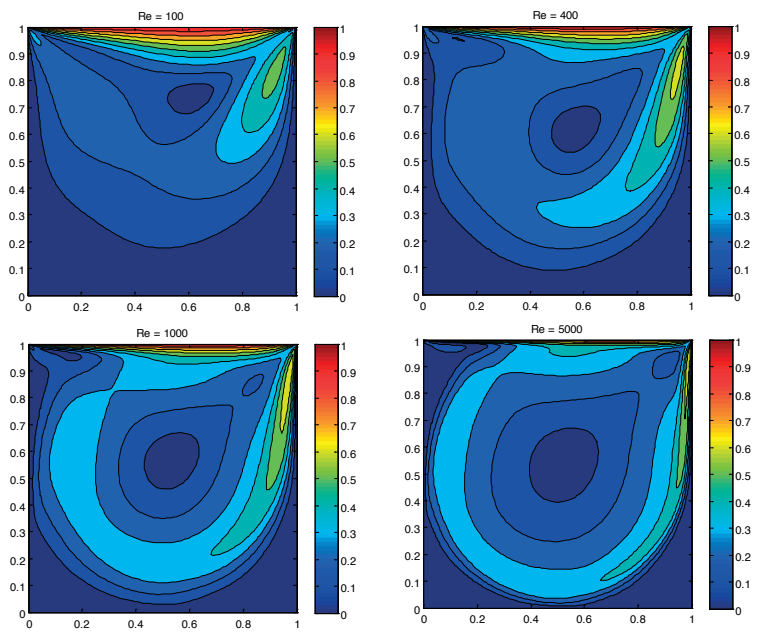

Fig. 6 Velocity magnitude for different Reynolds numbers

solution of the lid-driven cavity flow problem for various meshes and Reynolds numbers. The results demonstrate that the method is effective and useful forsolving this type of flow.

\section{References}

[1] NITHIARASU, P, ZIENKIEWICZ, O.: Analysis of an Explicit and Matrix Free Fractional Step Method for Incompressible Flows, Comput Methods Appl Mech Eng, 2006, 195:5537-51.

[2] LIN, C. Y., GU, M. H., YOUNG, D. L., SLADEK, J., SLADEK, V.: The Localized Method of Approximated Particular Solutions for Solving Two-Dimensional Incompressible Viscous Flow Field, Eng Anal Bound Elem, 2015, 57:23-36.

[3] KOVARIK, K.: Numerical Simulation of Groundwater Flow and Pollution Transport Using the Dual Reciprocity and RBF Method, Communications - Scientific Letters of the University of Zilina, No. 3a, 2010, pp.5-10, ISSN 1335-4205. 
[4] KOVARIK, K.: The New Numerical Methods for Mathematical Modelling of Pollutant Transport, Communications - Scientific Letters of the University of Zilina, No. 4, 1999, pp. 59-68, ISSN 1335-4205.

[5] ZHU, T., ZHANG, J., ATLURI, S.: A Meshless Numerical Method Based on the LocalBoundary Integral Equation (LBIE) to Solve Linear and Non-Linear BoundaryValue Problems. Eng Anal Bound Elem 1999, 23:375-89.

[6] SLADEK, V, SLADEK, J, ATLURI, S. N., VAN KEER, R.: Numerical Integration of Singularities in Meshless Implementation of Local Boundary Integral Equations. Comput Mech, 2000, 25:394-403.

[7] KOVARIK, K, MUZIK, J.: A Meshless Solution for Two Dimensional Density-Driven Groundwater Flow, Eng Anal Bound Elem, 2013, 37:187-96.

[8] GINGOLD, R. A., MONAGHAN, J. J.: Smoothed Particle Hydrodynamics: Theory and Applications to Non-Spherical Stars, Mon Not R Astron Soc, 1977, 181:375-89.

[9] KANSA, E. J.: Multiquadrics- a Scattered Data Approximation Scheme with Application to Computational Fluid Dynamics, Comput Math Appl, 1990, 19:127-45.

[10] SANYASIRAJU, Y, CHANDHINI, G.: Local Radial Basis Function Based Gridfree Scheme for Unsteady Incompressible Viscous Flows, J. Comput Phys, 2008, 227:8922-48.

[11] SHU, C, DING, H, YEO, K. S.: Local Radial Basis Function-Based Differential Quadrature Method and its Application to Solve Two-Dimensional Incompressible Navier-Stokes Equations, Comput Methods Appl Mech Eng, 2003, 192:941-54.

[12] ATLURI, S. N., KIM, H-G., CHO, J. Y.: A Critical Assessment of the Truly Meshless Local Petrov-Galerkin (MLPG), and Local Boundary Integral Equation (LBIE) Methods, Comput Mech, 1999, 24:348-72.

[13] Lin, H., ATLURI, S. N.: The Meshless Local Petrov-Galerkin (MLPG) Method for Solving Incompressible Navier-Stokes Equations, Comput Model Eng Sci, 2001, 2:117-42.

[14] GHIA, U., GHIA, K. N., SHIN, C. T.: High-resolutionsfor Incompressible Flow Using the Navier-Stokes Equations and a Multigrid Method, J. Comput Phys. 1982, 48:387-411.

[15] KOVARIK, K, MUZIK, J, MAHMOOD, M. S.: A Meshless Solution of Two Dimensional Unsteady Flow, Eng Anal Bound Elem, 2012, 36:738-43.

[16] NAJAFI, M., AREFMANESH, A., EJILELA, V.: Meshless Local Petrov-Galerkin Method Higher Reynolds Numbers Fluid Flow Applications, Eng Anal Bound Elem, 2012, 36:1671-85.

[17] HARDY, R. L.: Theory and Applications of the Multiquadrics-Biharmonic Method (20 years of discovery 1968-1988), Comput Math Appl, 1990, 19:163-208. 